\title{
Particle Size Distribution of Aromatic Incense Burning Products
}

\author{
Hsin-Yi Lin, Der-Jen Hsu, and Jia-Shan Su
}

\begin{abstract}
Aromatic incense burning, which is aimed to alleviate some psychological and physical disorders, in indoor settings has been popular globally, yet the resulting indoor air pollution and the potential hazards arisen from incense burning has usually been ignored by most people. To date, knowledge about aromatic incense burning and the potential adverse health effects are still limited. Whether the size distribution of particulate form of combustion products is determined by the fragrance of aromatic incense burning is not clear. Accordingly, the present study is aiming to explore the size distribution of the particulate products from aromatic incense burning. In this study, three kinds of aromatic incense (lavender, rose, aglaia) were combusted in a homemade chamber, the Marple cascade impactor was used to collect different size ranges of particles and other combustion-related pollutants. The results of the study indicated that most of the particles emitted were smaller than $\mathbf{P M}_{2.5}$, regardless the fragrance of aromatic incense. The major sizes fell within the range between $1.55 \mu \mathrm{m}$ and $0.93 \mu \mathrm{m}$ and lavender incense was found to have the greatest emission factor in this size range. Compared with the regular incenses, aromatic incense burning has smaller emission factor. This study concludes that the combustion products of these three fragrances of aromatic incense are mainly $\mathbf{P M}_{2.5}$ and may pose adverse health effect when inhaled. Therefore, adequate ventilation is strongly suggested when aromatic incense is burning and moderate usage is advised. Moreover, future studies on other popular fragrances of incense and many more forms of aromatic products are warranted.
\end{abstract} $\mathbf{P M}_{2.5}$.

Index Terms-Aromatic incense, particle size distribution,

\section{INTRODUCTION}

Gods and ancestors worshipping by burning incense sticks are quite common in many Asian countries. Those incense sticks are usually burned in indoor environments such as temples and household, where poorly ventilated condition is usually observed. One study showed that incense burning for worship purposes is found in $50 \%$ of the Taiwanese household [1]. This indicates that the majority of the Taiwanese are used to smoke dusts resulted from incense burning in their houses during their life time. However, many studies also indicated incense burning may result in

Manuscript received September 15, 2014; revised January 15, 2015.

Hsin-Yi Lin is with Chang Jung Christian University, Department of Occupational Safety and Health, Tainan, Taiwan (e-mail: linone@mail.cjcu.edu.tw).

Der-Jen Hsu is with National Kaohsiung First University of Science and Technology, Department of Safety, Health and Environmental Engineering, Kaohisung, Taiwan (e-mail: hsuderjen@nkfust.edu.tw).

Jia-Shan $\mathrm{Su}$ is with Unimicron Technology Corporation, Taoyuan, Taiwan (e-mail: suzanne@gmai.com). the production of airborne particles and carcinogens, such as polycyclic aromatic hydrocarbons (PAHs), which may cause adverse health effects [2]. There are, at least, 30 types of PAHs are considered as carcinogens by IARC [3]. Chao and his colleagues also pointed out that PAHs from the products of incense burning may induce the human lung cancers [4]. In addition to carcinogenic effects, exposure to such pollutants may result in some acute irritation symptoms, especially for the upper airway [1]. In fact, incense burning has been considered as a significant contributor of indoor particle concentration in Asian countries [5]. Studies have shown that the solid-phased and gaseous-phased products from incense burning are one of the major sources of indoor air pollution and are hazardous to the human health. Moreover, the particulates from incense burning are within the size range of $\mathrm{PM}_{2.5}$, which can reach the human alveolar region easily, may pose adverse health effects [6]. Given the abovementioned health effects, children before school age and the elderly are the highest risk group because such group of people spends longer time at homes than other groups.

Beside the incense burning for worship purposes, incenses with the addition of essential oils has become popular and commercially available in the past decade. Aromatic incense burning has commonly seen in many indoor environmental settings, such as offices and households. Not only providing fragrance in indoor environments, different fragrances of aromatic incense are available for customers for the purposes of alleviating some psychological and physical disorders, such as sleep inducing, calming and stress relief. Similar to the traditional incense sticks, aromatic incense burning also produce large quantities of airborne particles. Since many studies have confirmed that use of essential oils would generate various types of volatile organic compounds (VOCs) and formaldehyde [7], [8]. It is, thus, reasonable to hypothesize that pollutants generated by burning aromatic incenses may possibly differ from burning traditional incense sticks in quantity and quality as well. Unfortunately, those pollutants from such aromatic incense burning in indoor environments and their health effects have not been thoroughly investigated. Moreover, since various aromatic incenses are commercially available, whether the type of pollutants and the size distribution of particles emitted vary with different fragrances (i.e., essential oils from different herbs or plants) of aromatic incense burning and thus determine the health effects is not completely understood at this stage. Accordingly, the objective of this study is to investigate the size distribution of particles from burning three chosen fragrances of aromatic incense. Emission factor of such particulate products from burning was also presented and discussed. 


\section{METHODS AND MATERIALS}

\section{A. Selection of Aromatic Incense}

Various fragrances of aromatic incense are commercially available in the markets. Three best-sold fragrances of aromatic incense were selected based on our market survey in Tainan and Kaohsiung area of Taiwan, including lavender (Lavandula angustifolis), rose (Rosa multiflora) and aglaia (Aglaia odorata). Considering the length of incense stick, the above three fragrances of aromatic incense were from the same manufacturer and were in the same length (about $15 \mathrm{~cm}$ ). To calculate the emission factor of incense burning, each stick of aromatic incense was weighted before burning. The time period for burning these aromatic incense sticks was approximately 35 minutes.

\section{B. Air Sampling}

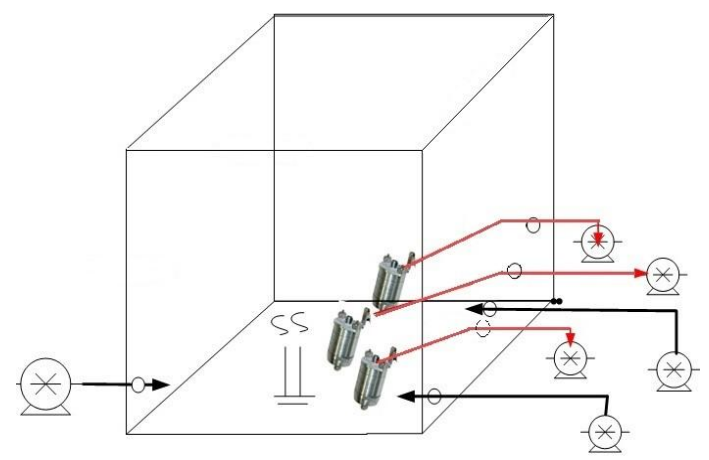

Fig. 1. Experimental setup for sizing particulate matter resulting from aromatic incense burning products.

The size distribution of the particulate form of incense burning products was determined by the Marple personal cascade impactor (Marple 298, New Star Environmental Inc. Roswell, GA, USA) located in a homemade acrylic box $(60 \times 60 \times 60 \mathrm{~cm})$ where aromatic incense was burned. The box was ventilated by two pumps, one flows in and the other flows out, in the flow rate of 2.5 liters per minute to maintain adequate air circulation in the burning chamber. For each fragrance, two sticks of aromatic incense were burned when air sampling was conducted by three Marple cascade impactors, each in the flow rate of 2 liters per minute by a SKC Model 222 sampling pump (SKC Inc., Pittsburgh, Pennsylvania, USA). The experimental setup is showed in Fig. 1. Particle mass for the specific size range was determined by the difference between pre- and post-weighed mass of filter paper in the corresponding stage by using a precision balance (Mettler-Toledo Inc., Zurich, Switzerland). All filer papers were pre-conditioned before pre-weighed and post-conditioned before post-weighed in a moisture buster maintained in constant temperature and relative humidity to avoid any possible error resulting from moisture.

\section{RESULTS AND DISCUSSION}

The cut-point size for each stage of Marple 298 is 21.3, $14.8,9.8,6.0,3.5,1.55,0.93$ and $0.52 \mu \mathrm{m}$, respectively. The results showed that the gained weight from the filters collecting particle sizes larger than $3.5 \mu \mathrm{m}$ was very limited, this is particularly true for particles larger than $9.8 \mu \mathrm{m}$. Among all size ranges, particles ranging from 0.93 to $1.55 \mu \mathrm{m}$ have the largest percentage, followed by the ranges of $0.52 \sim 0.93 \mu \mathrm{m}$ and $1.55 \sim 3.5 \mu \mathrm{m}$. Therefore, particles larger than $9.8 \mu \mathrm{m}$ were not considered and the size distribution for the rest of the particulate products from three fragrances of aromatic incenses burning was presented as $\mathrm{PM}_{2.5}, \mathrm{PM}_{2.5 \sim 10}$ and $\mathrm{PM}_{10}$ based on the gain weight from the filter of the corresponding stage. Table I shows the average weight (standard deviation) and percentage of particulate products from burning lavender, rose and aglaia incense based on the three Marple impactors. Obviously, the majority of the particulate products from aromatic incense burning are categorized as $\mathrm{PM}_{2.5}$. Particulate combustion products larger than $2.5 \mu \mathrm{m}$ was negligible and those larger than $10 \mu \mathrm{m}$ were even not detected in rose and aglaia incenses. Such finding in size range is consistent with those from incense burning for worshipping ancestors and gods in the literature [2], [4], [6], [9], [10]. Among the three fragrances tested, lavender incense burning generated much more amount of particles than rose and aglaia incenses.

TABLE I: The Weight AND PERCENTAge of PARTICUlate Products From Three ARomatic INCENSE BURNING

\begin{tabular}{|c|c|c|c|}
\hline \multicolumn{4}{|c|}{ Type of aromatic incense } \\
\hline \multirow{2}{*}{ Size distribution } & Lavender & Rose & Aglaia \\
\hline \multirow{2}{*}{$>\mathrm{PM}_{10}$} & $\begin{array}{c}\text { Weight }( \pm \text { s.d. })(\mathrm{mg}) \\
(\%)\end{array}$ & $\begin{array}{c}\text { Weight }( \pm \text { s.d.) }(\mathrm{mg}) \\
(\%)\end{array}$ & $\begin{array}{c}\text { Weight }( \pm \text { s.d.) }(\mathrm{mg}) \\
(\%)\end{array}$ \\
\hline \multirow{2}{*}{$\mathrm{PM}_{2.5} \sim \mathrm{PM}_{10}$} & $0.567( \pm 0.115)$ & N.D. & N.D. \\
& $0.545 \%$ & N.D. & $0.733( \pm 0.462)$ \\
\multirow{2}{*}{$\mathrm{PM}_{2.5}$} & $1.07( \pm 0.321)$ & N.D. & $0.496 \%$ \\
\hline \multirow{2}{*}{ Total } & $1.03 \%$ & $64.5( \pm 6.92)$ & $46.4( \pm 6.7)$ \\
& $92( \pm 14.6)$ & $100 \%$ & $98.5 \%$ \\
\hline & $104( \pm 14.6)$ & $64.5( \pm 8.16)$ & $47.2( \pm 6.24)$ \\
& $100 \%$ & $100 \%$ & $100 \%$ \\
\hline
\end{tabular}

The emission factor of particulate products from incense burning is expressed as the ratio of particle mass generated to each gram of incense burned. Fig. 2 shows the emission factors of three aromatic incense burning based on the size range. Apparently, lavender incense was with the greatest emission factor as expected. As shown in the figure, the size range between $1.55 \sim 0.93 \mu \mathrm{m}$ was found to have the greatest emission factor and that of size range between $0.93 \sim 0.52$ $\mu \mathrm{m}$ followed, regardless of aromatic fragrance. Additionally, the emission factor in this size range for lavender incense is approximately two folds greater than that from aglaia incense. Comparing the particulate emission factor of aromatic incense with the regular incense found that the former is smaller than the latter [2]. The fact of higher emission factor for burning regular incense was also found in the work of Richard et al. [11]. Other than the essential 
oils added in the aromatic incense, the difference between regular incense for worshipping and aromatic one is its length, consequently the burning duration. The aromatic incense burned in the current study was about $15 \mathrm{~cm}$ in length, while the regular incense was about $40 \mathrm{~cm}$ long. There are several possible explanations for such discrepancy in emission factor. One of them is that more amount of particles tend to be generated during longer combustion period and this provides higher frequency of collision and greater chances of agglomeration in a confined chamber. Therefore, larger quantity of particle is collected as a result of burning longer incenses. The other possible explanation might be the dimension and ventilation condition of the combustion chamber used in each study group. Such difference is likely to affect how the generated particles were dispersed and sampled. Therefore, the comparison was not made on a solid basis, due to the different experimental conditions. In addition to the above-mentioned factor, the essential oil added while aromatic incenses were manufactured might play an important but unknown role. Such conjecture is grounded in the finding that incenses with lavender essential oil were with 2-fold of emission factor greater than the incenses with other essential oils, such as aglaia. Though not known, the chemical compounds in the essential oils mixing with the ingredients of incense might affect the forms of combustion products distribute in the air, i.e., solid or gaseous forms. However, this issue is beyond the scope of the current study and further researches in the future are needed for confirmation.

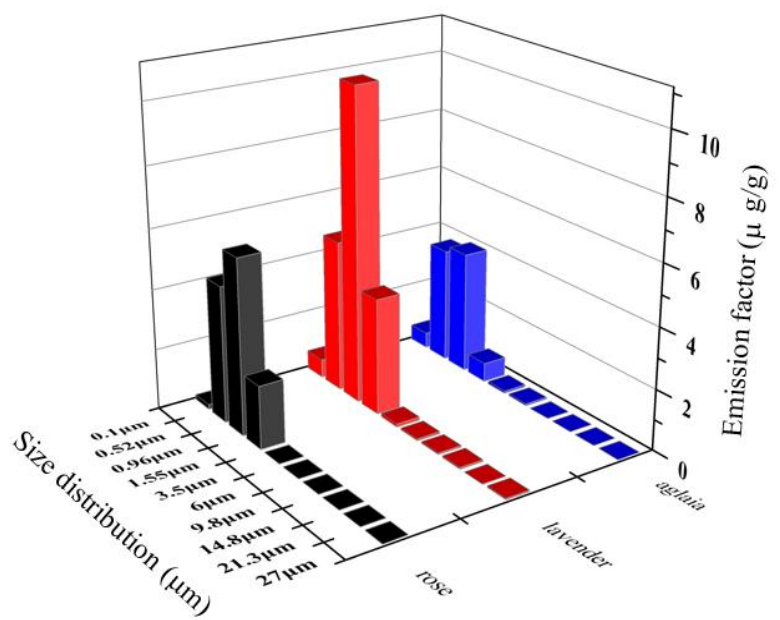

Fig. 2. Emission factor of different particle sizes from three types of aromatic incense burning.

This study indicated that the emission factor of particulate from burning aromatic incense varies with the fragrance, especially in the size range of the most hazardous to the human respiratory tract (i.e., $\mathrm{PM}_{2.5}$ ). This also implies that the health risk resulted from burning different fragrances of aromatic incense in the indoor settings varies as well. Since there are various fragrances of aromatic incense available in the markets, such as eucalyptus (Eucalyptus globules), tea tree (Melaleuca alternifolia) and lemon (Citrus limon), investigations of those commonly seen and equally favorable fragrances of aromatic incense seem to be warranted, due to the finding of the current study. Moreover, lavender incense was found to have the greatest emission factor of $\mathrm{PM}_{2.5}$ among the three chosen fragrances. Yet, lavender incense was also most favored by the customers in Taiwan. Obviously, such information should be made available for the customers or public from the viewpoint of reducing health risk from the exposure to aromatic incense burning products.

Several studies involving the use of essential oils have been conducted in the indoor environments [7], [8], [12]. Those studies all pointed out that some compounds in essential oils, such as terpenes containing unsaturated double bonds, contribute the formation of formaldehyde and nano-sized secondary organic aerosols (SOAs) through the reaction of terpenes with indoor background level of ozone. As essential oils extracted from plants were added to manufacture different fragrances of aromatic incenses, some VOCs in the essential oils must have been evaporated during incense burning. Some VOCs with unsaturated double bonds may react with indoor background ozone and form SOAs. Among the three fragrances selected in the current study, lavender was confirmed to contain terpenes such as $\mathrm{d}$-limonene, $\alpha$-pinene and $\beta$-pinene, which Hoffmann and his coworkers identified as SOAs precursors [13]. Given such possible chemistry in our combustion chamber, there might be two different sources of the particles collected by the Marple impactors, i.e., micro-sized combustion products and nano-sized SOAs. Though it is not capable to distinguish the contribution of the two sources for the samplers used in this study, the authors presume that combustion products outweigh the SOAs from the perspective of particle mass. Considering the mass of nano-sized SOAs, this seems to be a solid presumption. On the contrary, SOAs might outweigh the combustion products in view of particle number concentration. Therefore, particulate burning products of aromatic incense, again, leads to a long-time controversial issue, i.e., whether mass concentration or number concentration counts in view of the adverse human health effects?

The findings of this study have shed light on some interesting and important facts about the particulate products of aromatic incense burning, though many mechanisms behind the formation of particulate products and the distribution of solid and gaseous forms of products still remain unknown. Given the finding in this work, the authors suggest that future studies should carry out experiments on burning products of regular and aromatic incense burning under the same condition, so that a reasonable and solid comparison can be made to understand the possible discrepancy of the two. PAHs emission from both types of incense is another important topic to be studied. How essential oils, which are added in the aromatic incense, affect the forms of burning products of aromatic incense and whether the SOAs precursors in the essential oils contribute the formation of SOAs while aromatic incenses are burning are also the future topics of importance.

Some precautions should be taken to reduce the health risk of people who are in the indoor settings where aromatic incenses are burned. Feasible approaches include increasing of ventilation to dilute particle concentration, periodic cleaning or replacement of HVAC facilities as well as using non-ozone-emitting air cleaners and prohibition the use of laser printers or photocopy machines (i.e., sources of ozone emission) while burning aromatic incenses for prevention of 
indoor air chemistry between ozone and SOAs precursors.

\section{CONCLUSIONS}

This study concludes that $\mathrm{PM}_{2.5}$ contributes the major part of particulate matter emitted from aromatic incense burning, regardless of aromatic fragrances. In terms of size range of particulate form of burning pollutants, aromatic incense and traditional incense sticks for worshipping ancestors and gods are alike. Among the fragrances tested, lavender incense was found to have the greatest emission factor for the particle size ranging between 1.55 and $0.93 \mu \mathrm{m}$. This implies that different fragrances of aromatic incense generate different amount of particulate matter, and consequently may pose different levels of health risk to those exposed. The emission factor of the aromatic incense found in the current study was all lower than that obtained from the regular incense sticks for worshipping ancestors and gods. To reduce the health risk of people who are exposed to incense burning products, the authors suggest that adequate ventilation is needed when aromatic incense is burning and moderate usage is also advised. For the perspective of public health protection, more research efforts should be made to the understanding of the products resulting from aromatic incense burning. SOAs resulted from the indoor air chemistry between ozone and evaporated essential oils in aromatic incenses is also an important topic to be studied in the future.

\section{ACKNOWLEDGEMENTS}

The authors are in great debt to Drs. Wen-Jhy Lee and Yu-Cheng Chang at Department of Environmental Engineering, National Cheng Kung University, Tainan, Taiwan, Dr. Chi-Ru Yang at Department of Environmental Engineering and Science, Chia-Nan University of Pharmacy and Science, Tainan, Taiwan, Dr. How-Ran Chao at Department of Environmental Science and Engineering National Pingtung University of Science and Technology Pingtung, Taiwan for the help in sampling activities and consultation.

\section{REFERENCES}

[1] K. C. Chiang, C. P. Chio, Y. H. Chiang, and C. H. Liao, "Assessing hazardous risks of human exposure to temple airborne polycyclic aromatic hydrocarbons," Journal of Hazardous Materials, vol. 166, pp. 676-685, 2009.

[2] C. R. Yang, T. C. Lin, and F. H. Chang, "Particle size distribution and PAH concentrations of incense smoke in a combustion chamber," Environmental Pollution, vol. 145, pp. 606-615, 2007.

[3] P. Navasumrit, M. Arayasiri, O. M. T. Hiang et al., "Potential health effects of exposure to carcinogenic compounds in incense smoke in temple workers," Chemico-Biological Interactions, vol. 173, pp. 19-31, 2008

[4] H. R. Chao, T. C. Lin, and J. H. Hsieh, "Composition and characteristics of PAH emission from Taiwanese temples," Journal of Aerosol Science, vol. 28, pp. S303-S304, 1997.

[5] C. S. Li and Y. S. Ro, "Indoor characteristics of polycyclic aromatic hydrocarbons in the urban atmosphere of Taipei," Atmospheric Environment, vol. 34, pp. 611-620, 2000.
[6] S. W. See and R. Balasubramanian, "Characterization of fine particle emissions from incense burning," Building and Environment, vol. 46, pp. 1074-1080, 2011.

[7] D. J Hsu, H. L, Huang, and S. C. Sheu, "Characteristics of air pollutants and assessment of potential exposure in SPA centers during aromatherapy," Environmental Engineering Science, vol. 29, pp. 79-85, 2012.

[8] H. L. Huang, T. J. Tsai, N. Y. Hsu, C. C. Lee, P. C. Wu, and H. J. Su, "Effects of essential oils on the formation of formaldehyde and secondary organic aerosols in an aromatherapy environment," Building and Environment, vol. 57, pp. 120-125, 2012.

[9] G. C. Fang, C. N. Chang, Y. S. Wu, C. J. Yang, S. C. Chang, and I. L. Yang, "Suspended particulate variations and mass size distributions of incense burning at Tzu Yun Yen temple in Taiwan, Taichung," Science of the Total Environment, vol. 299, pp. 79-87, 2002.

[10] Y. S. Cheng, W. E. Bechtold, C. C. Yu, and I. F. Hung, "Incense smoke: characterization and dynamic in indoor environments," Aerosol Science and Technology, vol. 23, pp. 271-281, 1995.

[11] C. M. Richard, P. N. Khanh, W. T. Eric, E. H. Esther, and F. P. Robert, "Phyical characterization of incense aerosols," Science of the Total Environment, vol. 193, pp. 149-158, 1996.

[12] H. J. Su, C. J. Chao, H. Y. Chang, and W. C. Wu, "The effects of evaporting essential oils on indoor air quality," Atmospheric Environment, vol. 41, pp. 1230-1236, 2007.

[13] T. Hoffmann, J. R. Odum, F. Bowman, D. Collins, D. Klockow, R. C. Flagan, and J. H. Seinfeld, "Formation of organic aerosols from the oxidation of biogenic hydrocarbons," Journal of Atmospheric. Chemistry, vol. 26, pp. 189-222, 1997.

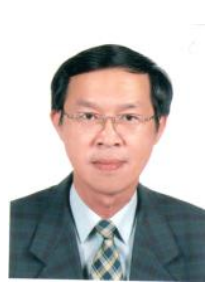

Hsin-Yi Lin obtained his B.S. degree in environmental engineering from National Chung-Hsin University, Taiwan in 1985; M.S. degree and PhD degree in civil engineering from Central University, Taiwan in 1985 and 1993, respectively. He is currently an associated professor in Chang-Jung Christmas University, Department of Occupational Safety and Health. Dr. Lin's research interests include indoor air quality, waste water treatment and solid waste management.

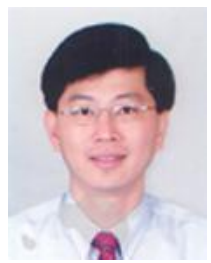

Der-Jen Hsu received his B.S. degree in forestry from National Taiwan University in 1986 and M.S. degree in environmental sciences from Rutgers University, New Jersey, US in 1991 and Ph.D degree in environmental health sciences from The Johns Hopkins University, Maryland, US in 1996. He is currently a professor in the Department of Safety, Health and Environmental Engineering, National Kaohsiung First University of Science and Technology, Kaohsiung, Taiwan. Dr. Hsu's research interests include indoor air quality, occupational hygiene, human lung particle desposition and human factor engineering. In the field of indoor air quality, his publication includes emiited BTEX and resulting SOA from photocope machines, indoor air quality in long-distance buses as well as VOCs and SOA during aromatherapy in spa centers in Taiwan. As the corresponding author of this work, Dr. Hsu currently is continuing his work in studying the PAHs emitted from the aromatic incense burning.

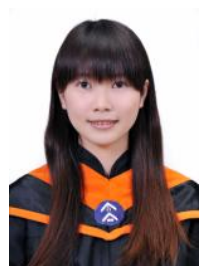

Jia-Shan Su received her B.S. degree in occupational safety and health in Chang-Jung Christian University in 2010 and M.S. degree in occupational health from National Kaohsiung First University of Science and Technology in 2012. She is currrently working as an industrial hygienist at Unimicron Technology Corporation, Taoyuan, Taiwan. 